\title{
Laser interferometry for the Big Bang Observer
}

\author{
Gregory M Harry ${ }^{1}$, Peter Fritschel ${ }^{1}$, Daniel A Shaddock ${ }^{2}$, \\ William Folkner ${ }^{2}$ and E Sterl Phinney ${ }^{3}$ \\ ${ }^{1}$ LIGO Laboratory, Massachusetts Institute of Technology, NW17-161, Cambridge, MA 02139, \\ USA \\ 2 Jet Propulsion Laboratory, California Institute of Technology, Pasadena, CA 91109, USA \\ ${ }^{3}$ California Institute of Technology, Pasadena, CA 91125, USA \\ E-mail: gharry@ligo.mit.edu
}

Received 24 January 2006, in final form 7 June 2006

Published 7 July 2006

Online at stacks.iop.org/CQG/23/4887

\begin{abstract}
The Big Bang Observer is a proposed space-based gravitational-wave detector intended as a follow on mission to the Laser Interferometer Space Antenna (LISA). It is designed to detect the stochastic background of gravitational waves from the early universe. We discuss how the interferometry can be arranged between three spacecraft for this mission and what research and development on key technologies are necessary to realize this scheme.
\end{abstract}

PACS numbers: $95.55 . \mathrm{Ym}, 04.80 . \mathrm{Nn}, 95.55 . \mathrm{Br}$, 95.75.Kk

(Some figures in this article are in colour only in the electronic version)

The Big Bang Observer (BBO) is proposed to NASA as a Beyond Einstein mission [1] targeted at detecting stochastic gravitational waves from the very early universe. It will also be sensitive to the final year of binary compact body (neutron stars and stellar mass black holes) inspirals out to $z<8$, mergers of intermediate mass black holes at any $z$, rapidly rotating white dwarf explosions from Type 1a supernovas at distances less than $1 \mathrm{Mpc}$ and $\sim 1 \mathrm{~Hz}$ pulsars with nonaxisymmetric magnetic fields of $B>3 \times 10^{14} \mathrm{G}$. More detailed discussion of sources for BBO can be found in a recent paper [2]. The mission is proposed in a number of stages, the first of which will consist of three spacecraft in solar orbit separated from each other by 50000 kilometres. Ultimately, in the final stage, there would be three such constellations of spacecraft, separated by $120^{\circ}$ from each other in solar orbit. The shorter arm length than the $5 \times 10^{6} \mathrm{~km}$ arms planned for the Laser Interferometer Space Antenna (LISA) means the most sensitive bandwidth will be higher, between 0.1 and $1 \mathrm{~Hz}$. This will give sensitivity to gravitational waves in the frequency band between LISA [3] (from $10 \mu \mathrm{Hz}$ to $10 \mathrm{mHz}$ ) and the ground-based advanced LIGO [4] (Laser Interferometer Gravitational-Wave Observatory) (from 10 to $3000 \mathrm{~Hz}$ ). The sensitivity near $0.5 \mathrm{~Hz}$ will be about $1 \times 10^{-24} \mathrm{~Hz}^{-1 / 2}$ in strain or $5 \times 10^{-17} \mathrm{~m} \mathrm{~Hz}^{-1 / 2}$ in displacement. This is compared to $1 \times 10^{-20} \mathrm{~Hz}^{-1 / 2}$ and 


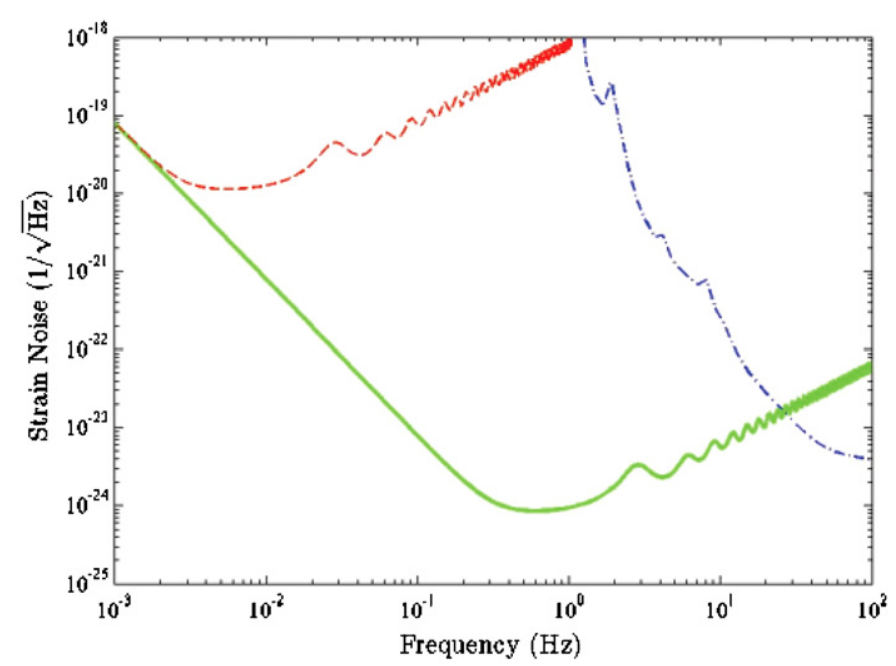

Figure 1. Strain noise, as the square root of strain power spectral density, versus frequency for three advanced interferometric gravitational-wave detectors: LISA, Advanced LIGO and BBO. LISA is the dashed (red) curve, Advanced LIGO is the dash-dotted (blue) curve and BBO is the solid (green) curve. In all three cases, the noise has been averaged over all possible source locations on the sky and all source polarizations.

$5 \times 10^{-11} \mathrm{~m} \mathrm{~Hz}^{-1 / 2}$ for LISA. The noise spectra of BBO, LISA and Advanced LIGO are compared in figure 1 . In this paper, we explain how the interferometry can be arranged to achieve this level of sensitivity.

Shot noise from the laser will be a limiting noise source given by

$$
S_{x}(f)=2 h c \lambda^{3} L^{2} /\left(2 \pi^{4} \eta P D^{2} w^{2}\right),
$$

where $h$ is Planck's constant, $c$ is the speed of light, $\lambda$ is the wavelength of light, $L$ is the spacecraft separation, $\eta$ is the optical loss in the optics including the photodiode quantum efficiency, $P$ is the laser power, $D$ is the collection mirror diameter and $w$ is the beam waist radius. To reach the required level of sensitivity, the laser power must be high and/or the wavelength must be kept small. Each spacecraft will have two $300 \mathrm{~W}$ lasers with a wavelength $355 \mathrm{~nm}$, obtained by frequency tripling Nd:YAG lasers. About $8 \mathrm{~W}$ will be detected at the far spacecraft after diffraction losses from a $2.5 \mathrm{~m}$ diameter collecting mirror on the far spacecraft, $5 \times 10^{7} \mathrm{~m}$ away. The laser light monitors the position of free-falling test masses to detect displacement caused by gravitational waves. The length between the test masses will be controlled by applying forces on the masses to keep the photodiode signal locked on a dark fringe. This makes BBO more like a ground-based interferometer such as LIGO $[5,4]$ than LISA. LISA employs heterodyne interferometry based on offset lasers, and thus has no 'dark-fringe' position. LISA photodiodes are required to detect a full fringe of two interfered beams which will be scanned over many times a second. This limits the achievable noise in the photodiodes and so a similar scheme would not be possible for BBO with two $8 \mathrm{~W}$ beams interfered at each photodiode. Phase modulation of the BBO laser and subsequent demodulation will be used to extract a linear signal at the photodiode. Keeping this signal at a dark fringe will then reduce the power handling requirement of the photodiode.

A proposed layout of lasers and photodiodes on a spacecraft is shown in figure 2. Two lasers come onto each spacecraft which are used to both illuminate the arms between spacecraft and to monitor the position of the test mass relative to the local spacecraft. The arm between 


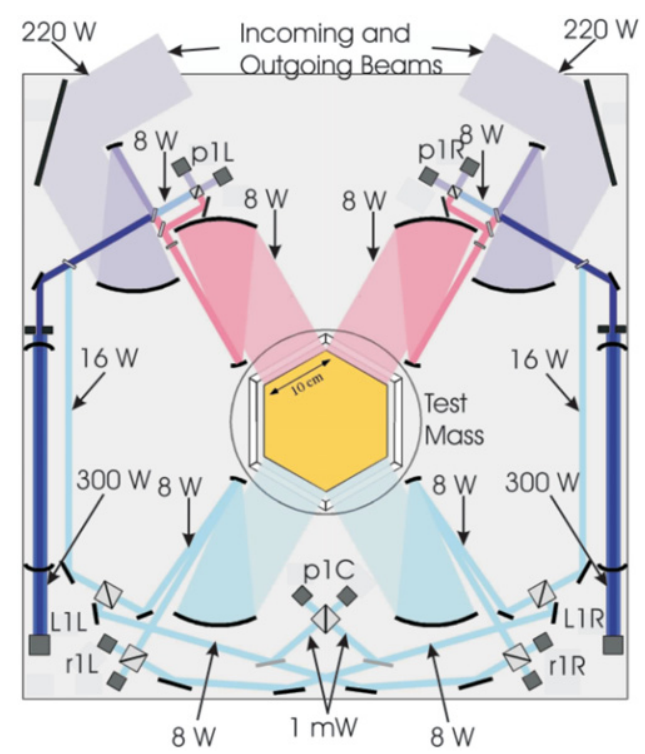

Figure 2. Layout of optics and photodiodes on spacecraft 1. Two laser beams come onto this bench at $300 \mathrm{~W}$ apiece. They are then each split into a $16 \mathrm{~W}$ beam, used for local sensing of the test mass-spacecraft separation, an $8 \mathrm{~W}$ beam, used to interfere with the incoming beam, and a $220 \mathrm{~W}$ beam (accounting for losses in the optics) which is sent to another spacecraft. The interference of beams is actually made with two photodiodes so that all of the light from both beams contributes to the signal. An additional mirror, not shown, redirects the outgoing beams so that light from L1L goes to the left and from L1R goes to the right.
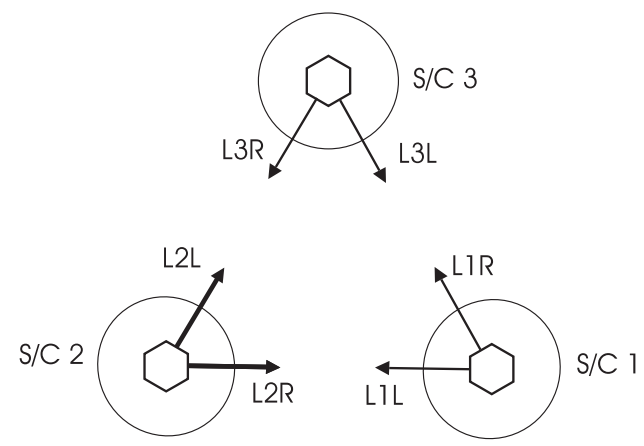

Figure 3. Constellation of all three spacecraft. The individual spacecraft are separated by $5 \times 10^{7} \mathrm{~m}$.

spacecraft 1 and 3 will be used as a stable frequency reference. A master laser on spacecraft 1 will be phase locked to this arm by feeding back the signal from photodiode p1R to laser L1R. This signal comes from the interference of the master laser, L1R, with light in the arm coming from laser L3L on spacecraft 3 (see figure 3). The other laser on spacecraft 1, L1L, will be phase locked to the master laser L1R using feedback from p1C, where the two lasers are interfered. Test mass 1 will be controlled in the direction connecting spacecraft 1 and 2 using the signal from $\mathrm{p} 1 \mathrm{~L}$, which has the interference of $\mathrm{L} 1 \mathrm{~L}$ and $\mathrm{L} 2 \mathrm{R}$. On spacecraft 2, laser L2R will be locked to laser L1 L, and laser L2L locked to laser L3R. Test mass 2 could be 
Table 1. Laser interference detection by photodiodes. The signals from the photodiodes are then fed back to the recipients in the third column.

\begin{tabular}{|c|c|c|}
\hline Photodiode & Interfering lasers & Recipient of feedback \\
\hline $\mathrm{p} 1 \mathrm{R}$ & L1R-L3L ${ }^{a}$ & Laser L1R \\
\hline r1R & $\mathrm{L} 1 \mathrm{~L}-\mathrm{L} 1 \mathrm{R}^{\mathrm{a}}$ & Spacecraft 1 \\
\hline $\mathrm{p} 1 \mathrm{C}$ & L1R-L1L & Laser L1L \\
\hline r1L & L1R-L1L ${ }^{a}$ & Spacecraft 1 \\
\hline $\mathrm{p} 1 \mathrm{~L}$ & $\mathrm{~L} 1 \mathrm{~L}-\mathrm{L} 2 \mathrm{R}^{\mathrm{a}}$ & Test mass 1 in $1-2$ direction \\
\hline $\mathrm{p} 2 \mathrm{R}$ & $\mathrm{L} 2 \mathrm{R}-\mathrm{L} 1 \mathrm{~L}^{\mathrm{a}}$ & Laser L2R \\
\hline $\mathrm{r} 2 \mathrm{R}$ & $\mathrm{L} 2 \mathrm{~L}-\mathrm{L} 2 \mathrm{R}^{\mathrm{a}}$ & Spacecraft 2 \\
\hline $\mathrm{p} 2 \mathrm{C}$ & L2L-L2R & Free for additional use \\
\hline $\mathrm{r} 2 \mathrm{~L}$ & $\mathrm{~L} 2 \mathrm{R}-\mathrm{L} 2 \mathrm{~L}^{\mathrm{a}}$ & Spacecraft 2 \\
\hline $\mathrm{p} 2 \mathrm{~L}$ & $\mathrm{~L} 2 \mathrm{~L}-\mathrm{L} 3 \mathrm{R}^{\mathrm{a}}$ & Laser L2L \\
\hline $\mathrm{p} 3 \mathrm{R}$ & $\mathrm{L} 3 \mathrm{R}-\mathrm{L} 2 \mathrm{~L}^{\mathrm{a}}$ & Test mass 3 in $2-3$ direction \\
\hline $\mathrm{r} 3 \mathrm{R}$ & $\mathrm{L} 3 \mathrm{~L}-\mathrm{L} 3 \mathrm{R}^{\mathrm{a}}$ & Spacecraft 3 \\
\hline $\mathrm{p} 3 \mathrm{C}$ & L3L-L3R & Laser L3R \\
\hline r3L & $\mathrm{L} 3 \mathrm{R}-\mathrm{L} 3 \mathrm{~L}^{\mathrm{a}}$ & Spacecraft 3 \\
\hline $\mathrm{p} 3 \mathrm{~L}$ & $\mathrm{~L} 3 \mathrm{~L}-\mathrm{L} 1 \mathrm{R}^{\mathrm{a}}$ & Laser L3L \\
\hline
\end{tabular}

${ }^{a}$ Laser first bounces off of local test mass.

left uncontrolled or it could be actuated on tangentially in the direction parallel to arms 1-3 to provide a third Michelson or a Sagnac signal. The signal for this additional actuation would come from photodiode $\mathrm{p} 2 \mathrm{C}$. On spacecraft 3, laser L3L will be locked to laser L1R, and laser L3R will be locked to laser L3L. Test mass 3 will be controlled in the direction connecting spacecraft 2 and 3 using feedback from p3R where lasers L3R and L2L are interfered. A complete description of the control architecture is given in table 1 .

The main beam coming out of each laser will be about $300 \mathrm{~W}$. This will come from a different board, and be passed onto the board shown in figure 2, possibly by a fibre. The beam will then go through a Fabry-Perot cavity which will serve as a passive mode cleaner to stabilize the beam direction and as a reference for frequency stabilization. A cavity finesse of about 100 is anticipated to provide sufficient filtering, adequate shot-noise limited frequency stability and high transmission efficiency. About $10 \%$ of the light power entering the cavity is anticipated being lost here. Immediately after the cavity, an $8 \mathrm{~W}$ and a $16 \mathrm{~W}$ beam will be picked off, one being used to interfere with the incoming beam and the other to monitor the position of the test mass relative to the spacecraft. The outgoing beam will then be sent to a telescope that expands the beam size and sends it to another spacecraft. Another $10 \%$ of the laser power will be lost in these output optics, so about $220 \mathrm{~W}$ will be projected to the other spacecraft.

The incoming beam will be reflected off a receiving telescope, where its plane wave will be converted into an Airy disc. The optimum power transmission occurs when the beam waist is 0.466 times the telescope diameter [6]. The telescope mirrors will have a diameter of $2.5 \mathrm{~m}$, so two can fit within the $5 \mathrm{~m}$ diameter space in the launch vehicle, which makes the optimum beam waist $1.1 \mathrm{~m}$. The geometric loss from diffraction-limited propagation over the $5 \times$ $10^{7} \mathrm{~m}$, starting from a $1.1 \mathrm{~m}$ spot and being collected by a $2.5 \mathrm{~m}$ diameter mirror, will bring the power down to about $9 \mathrm{~W}$. Assuming another $10 \%$ loss in power from the collection optics, the beam that is reflected off the local test mass will be about $8 \mathrm{~W}$. This beam is then interfered with the $8 \mathrm{~W}$ bean picked off of the outgoing laser. 
The incoming beam of about $8 \mathrm{~W}$ will result in a dc pressure on the test mass of about $54 \mathrm{nN}$ per beam. The $16 \mathrm{~W}$ beam of light from each local laser will be directed to the back of the optical bench. This beam is split into three ways: two high power $(\sim 8 \mathrm{~W})$ and a low power $(\sim 1 \mathrm{~mW})$. The first $8 \mathrm{~W}$ beam is used to measure the spacecraft position relative to the test mass. This beam also serves to balance the radiation force from the incoming beam; by balancing the incoming and local beam power levels to a few per cent, the resultant static force on the test mass (which must be compensated by the test mass actuation) can be reduced to a few nano-Newtons. The $1 \mathrm{~mW}$ beam is interfered with the equivalent $1 \mathrm{~mW}$ beam from the other laser on the same spacecraft at photodiode $\mathrm{p} 1 \mathrm{C}$ in figure 2. This signal is used to lock the frequency of one laser to the other. The second $8 \mathrm{~W}$ beam is interfered with the beam reflected off of the other backside (at $60^{\circ}$ to the first) of the test mass from the other laser. The signals from these local measurements ( $\mathrm{r} 1 \mathrm{~L}$ and $\mathrm{r} 1 \mathrm{R}$ in figure 2 ) will be sent to the spacecraft to control the two separate degrees of freedom. The third linear degree of freedom, and the angular ones, will be controlled separately. All photodiodes will be quadrant diodes where the sum signal of all four quadrants will provide the primary signal, and differences between the quadrants can be used to measure angular degrees of freedom.

Though the four beams reflecting off the test mass will be balanced to greatly reduce the net static radiation force, the amplitude noise on the beams will not be correlated, resulting in (uncompensated) acceleration noise. The acceleration noise can be written as

$$
S_{a}(f)=8 S_{p}(f) /(m c)^{2},
$$

where $S_{p}(f)$ is the laser power noise, $m$ is the mass of the test mass and $c$ is the speed of light. Assuming a mass of $10 \mathrm{~kg}$, at a relative intensity noise level of $\sqrt{S_{p}(f)} / P=10^{-8} \mathrm{~Hz}^{-1 / 2}$ in each beam, each degree of freedom will have acceleration noise of

$$
S_{a}(f) \approx 1 \times 10^{-26} \mathrm{~m} \mathrm{~s}^{-2} \mathrm{~Hz}^{-1 / 2} .
$$

This will make it a significant contributor to the acceleration noise budget. A relative intensity noise of $10^{-8} \mathrm{~Hz}^{-1 / 2}$ at $10 \mathrm{~Hz}$ has been demonstrated [7], but with much higher noise, near $10^{-6} \mathrm{~Hz}^{-1 / 2}$, at $0.1 \mathrm{~Hz}$. Continuing work on intensity noise reduction will be necessary.

The laser light incoming to each spacecraft will be a plane wave because of the long distance the beam travels. The image reflected off of the receiving mirrors will be an Airy disc, which is to be interfered with the on-spacecraft Gaussian beam. The resulting contrast defect on the photodiode needs to be low to keep the detected power down. A value of $10^{-4}$ for contrast defect is a good goal, making the power on the diode about $2 \mathrm{~mW}$. It may be necessary to have multiple photodiodes, at least for $\mathrm{p} 1 \mathrm{~L}$ and $\mathrm{p} 1 \mathrm{R}$ and the equivalents on spacecraft 2 and 3. Contrast defect issues are being worked out for LISA so BBO can take advantage solutions found there. Further development in mirror shapes and photodiode power handling may still be necessary.

A limited amount of light will be collected by the telescope, so the shot-noise limit will be (assuming the optimum waist size of $0.446 \times D[3]$ )

$$
S_{x}(f)=h c \lambda^{3} L^{2} /\left(2 \pi^{2} \eta P D^{4}\right),
$$

with $\lambda=355 \mathrm{~nm}, P=220 \mathrm{~W}, L=5 \times 10^{7} \mathrm{~m}, D=2.5 \mathrm{~m}$ and $\eta=0.6$, a shot-noise limited position noise of $1.5 \times 10^{-17} \mathrm{~m} \mathrm{~Hz}^{-1 / 2}$ is achieved. To obtain a quantum efficiency of 0.6, the most attractive commercial photodiodes are the Series 7 Super UV diodes from Centrovision, Inc. [10]. These diodes come as large as $1 \mathrm{~cm}^{2}$ in active area, though only single element devices are currently available; quadrant versions would need to be developed. The continuous wave intensity limit for these devices is $10 \mathrm{~mW} \mathrm{~cm}^{-2}$ or $10 \mathrm{~mW} \mathrm{CW}$ for the largest model. The response at $355 \mathrm{~nm}$ is approximately $0.18 \mathrm{~A} \mathrm{~W}^{-1}$, which corresponds to a 
quantum efficiency of 0.6. Of course, even higher quantum efficiency would be advantageous, as it could reduce the laser power requirements.

The allowed level of laser frequency noise, $S_{\nu}$, depends on the imbalance in the lengths of the arms, with

$$
S_{x}(f)=\lambda^{2}(\Delta l)^{2} S_{v} / c^{2},
$$

where $\Delta l$ is the imbalance. The imbalance $\Delta l$ can be held to about $1 \mathrm{~m}$ using a radio link between the spacecraft. A radio link is required primarily to measure the solar plasma electron content effect, which would otherwise be a limiting noise source. With an imbalance of $1 \mathrm{~m}$, laser frequency noise would need to be below $10^{-3} \mathrm{~Hz} \mathrm{~Hz}^{-1 / 2}$, in order to be 10 times less than the shot-noise level of equation (4). Active stabilization to the FP cavity will be limited to about $0.3 \mathrm{~Hz} \mathrm{~Hz}^{-1 / 2}$ at $0.1 \mathrm{~Hz}$, depending on temperature and materials used for the cavity, due to thermal noise considerations [8]. Further reduction of frequency noise can be achieved by stabilizing it to a long arm, as proposed for LISA in a recent paper [9]. With a unity gain frequency of $10^{5} \mathrm{~Hz}$ for this stabilization step, a reduction of $10^{3}$ in frequency noise could be achieved at $0.1 \mathrm{~Hz}$. Of course, the arm length matching may be much better than $1 \mathrm{~m}$, allowing for much higher frequency noise. In any event, a combination of local stabilization to the FP cavities, stabilization to the long arms and the common mode rejection ratio should provide adequate means for controlling frequency noise. The shot noise in photodiode $\mathrm{p} 1 \mathrm{C}$ in figure 2 is given by

$$
S_{v}=2 h v f^{2} /(\eta P)
$$

Using $v=c / \lambda=8.5 \times 10^{14} \mathrm{~Hz}, f=0.5 \mathrm{~Hz}, \eta=0.6$ and $P=1 \mathrm{~mW}$ (assuming that the signal is held halfway between a bright and a dark fringe), $\sqrt{S_{v}} \approx 1 \times 10^{-8} \mathrm{~Hz} \mathrm{~Hz}^{-1 / 2}$. Therefore, shot noise will not limit the frequency noise in lasers locked to the main laser.

The signal detection will be made using RF phase modulation of the laser light and synchronous RF demodulation of the photodetector outputs. Each laser will be phase modulated at a similar, but unique frequency, probably of order $10 \mathrm{MHz}$. The modulation frequency must be high enough to be above technical noises, so that the laser light is shot-noise limited. On the other hand, limited response times of photodiodes argue in general for lower frequencies. Thus, the choice of modulation frequency will involve trade-offs between the laser, modulator and photodiode technologies. The relatively large photodiodes necessary for power handling, near $1 \mathrm{~cm}^{2}$, may have sufficient capacitance to limit the frequency of modulation, however $10 \mathrm{MHz}$ should be obtainable. With the FP filter/reference cavity on the optical bench, there are two strategies for applying the modulation. If the modulation is applied after the cavity, the full laser power $(\sim 300 \mathrm{~W})$ will need to be transmitted through an electro-optic modulator (EOM). Handling this much power in an EOM will require significant research and development. The modulation could also be applied earlier in the optical chain, before amplification stages, to reduce the amount of power the EOM must handle. This would require, however, that the modulation be passed through the FP cavity. This would mean no reduction of intensity noise can occur at this pre-mode cleaner. The laser would need to be shot-noise limited at $10 \mathrm{MHz}$, which may require significant research and development on laser technology.

Thermal noise from the test mass must be considered as a contributor to the measurement noise. The test mass material must be chosen to have low mechanical loss at the operating temperature, low magnetic susceptibility, low thermal expansion, as well as a practical method of constructing a $10 \mathrm{~kg}$ mass. Sapphire may be an attractive option, as it is known to have low mechanical loss over a wide temperature band [11]. Brownian thermal noise is given by

$$
S_{x}(f)=\frac{2 k_{B} T}{\pi^{3 / 2} f} \frac{\phi}{w Y},
$$


where $k_{B}$ is Boltzmann's constant, $T$ is the temperature, $\phi$ is the loss angle of the test mass material, $w$ is the beam spot size $\left(1 / e^{2}\right.$ power radius) and $Y$ is the Young's modulus of the test mass material. Sapphire has been seen to have $\phi$ as low as $5 \times 10^{-9}[11,12]$, making the Brownian thermal noise well below BBO sensitivity at $0.5 \mathrm{~Hz}$, about $4 \times 10^{-20} \mathrm{~m} \mathrm{~Hz}^{-1 / 2}$ with $w=3 \mathrm{~cm}$ and $Y=4 \times 10^{11} \mathrm{~Pa}$.

Sapphire also has significant noise from thermo-elastic damping, which is given by

$$
S_{x}(f)=\frac{8}{\sqrt{2 \pi}} \alpha^{2}\left(1+\sigma^{2}\right)^{2} \frac{k_{B} T^{2}}{(\rho C)^{2}} \frac{\kappa}{w^{3}} \frac{1}{(2 \pi f)^{2}},
$$

where $\alpha$ is the test mass thermal expansion coefficient, $\sigma$ is Poisson's ratio, $\rho$ is the test mass density, $C$ is the test mass specific heat per mass and $\kappa$ is the thermal conductivity. This noise is also below BBO sensitivity for sapphire, about $2.3 \times 10^{-18} \mathrm{~m} \mathrm{~Hz}^{-1 / 2}$ at $0.5 \mathrm{~Hz}$ for $\alpha=5.6 \times 10^{-6} \mathrm{~K}^{-1}, \sigma=0.23, \rho=4.0 \times 10^{3} \mathrm{~kg} \mathrm{~m}^{-3}, C=770 \mathrm{~J}(\mathrm{~kg} \mathrm{~K})^{-1}$ and $\kappa=$ $39 \mathrm{~J} \mathrm{~s}\left(\mathrm{~m} \mathrm{~K}^{-1}\right.$. Silicon may be another material to consider, as it has low mechanical loss [13] and is more readily available in large sizes. High thermal conductivity is desired to handle fluctuating thermal loads, but unfortunately a high $\kappa$ also means high thermo-elastic noise.

The optical coatings needed to make the test masses reflective will also contribute significantly to thermal noise. Dielectric materials such as silica and tantala typically have much lower mechanical loss [14] than metallic coatings. Displacement noise due to the optical coating thermal noise is estimated as

$$
S_{x}(f)=\frac{2 k_{B} T}{\pi^{3 / 2} f} \frac{2(1+\sigma)(1-2 \sigma) d \phi_{\text {coat }}}{\sqrt{2} w^{2} Y},
$$

where $\phi_{\text {coat }}$ is the mechanical loss angle of the coating and $d$ is the coating thickness. For typical dielectric amorphous oxides, such as silica and tantala, $\phi_{\text {coat }}=2 \times 10^{-4}, Y=1 \times 10^{11}$ $\mathrm{Pa}, \sigma=0.2$ and $d=2 \mu \mathrm{m}$, giving a coating Brownian thermal noise of $1.4 \times 10^{-19} \mathrm{~m} \mathrm{~Hz}^{-1 / 2}$. The level of optical absorption in these coatings at $355 \mathrm{~nm}$ may be an issue and would need to be researched. The noise from magnetic susceptibility in the dielectric coatings would also need to be determined.

For BBO to achieve the needed sensitivity to detect the relic stochastic background of gravitational waves, much technology research must be done between now and the 2025 target launch date. Successful development of suitable UV photodiodes, high power and reduced noise lasers, appropriate mirror shapes, high power transmission optics and low thermal noise materials is crucial.

\section{Acknowledgments}

We would like to thank David Ottaway for useful discussions. This work was done under NASA grant NNG04GJ65G. This paper has been assigned LIGO Document Number LIGOP050054-00-R.

\section{References}

[1] Phinney E S et al 2004 The Big Bang Observer: direct detection of gravitational waves from the birth of the universe to the present NASA Mission Concept Study

[2] Crowder J and Cornish N J 2005 Phys. Rev. D 72083005

[3] Bender P L et al 1998 LISA Pre-Phase A Report 2nd edn MPQ-233 ed K V Danzmann (Garching, Germany: Max Planck Institute for Quantum optics)

[4] Fritschel P 2003 Second generation instruments for the Laser Interferometer Gravitational Wave Observatory Gravitational-Wave Detection ed M Cruise and P Saulson Proc. SPIE 4856 282-91 
[5] Abbott B et al 2004 Detector description and performance for the first coincidence observations between LIGO and GEO Nucl. Instrum. Methods Phys. Res. A 517 154-79

[6] Danzmann K V (ed) 1998 LISA Pre-Phase A Report 2nd edn MPQ-233 (Garching, Germany: Max Planck Institute for Quantum Optics)

[7] Rollins J, Ottaway D, Zucker M and Weiss R 2004 Opt. Lett. 29 1876-8

[8] Numata K, Kemery A and Camp J 2004 Phys. Rev. Lett. 93250602

[9] Sheard B J, Gray M B, McClelland D E and Shaddock D A 2003 Phys. Lett. A 3209

[10] www.centrovision.com/7des.htm

[11] Braginsky V, Mitrofanov V and Panov V 1986 Systems with Small Dissipation (Chicago: University of Chicago Press)

[12] Rowan S, Cagnoli G, Sneddon P, Hough J, Route R, Gustafson E K, Fejer M M and Mitrofanov V 2000 Phys. Lett. A 2655

[13] Rowan S, Byer R L, Fejer M M, Route R K, Cagnoli G, Crooks G, Crooks D R M, Hough J, Sneddon P and Winkler W 2003 Gravitational Wave Detection (Proc. SPIE vol 4856) ed M Cruise and P Saulson (Bellingham, WA: SPIE)

[14] Penn S D et al 2003 Class. Quantum Grav. 202917 